\title{
SOME QUANTITATIVE CHARACTERIZATIONS OF CERTAIN SYMPLECTIC GROUPS OVER THE BINARY FIELD
}

\author{
M. Akbari and A. R. Moghaddamfar \\ Received: 2 December 2013; Revised: 1 April 2014 \\ Communicated by Arturo Magidin
}

\begin{abstract}
Given a finite group $G$, denote by $\mathrm{D}(G)$ the degree pattern of $G$ and by $\mathrm{OC}(G)$ the set of all order components of $G$. Denote by $h_{\mathrm{OD}}(G)$ (resp. $\left.h_{\mathrm{OC}}(G)\right)$ the number of isomorphism classes of finite groups $H$ satisfying conditions $|H|=|G|$ and $\mathrm{D}(H)=\mathrm{D}(G)$ (resp. $\mathrm{OC}(H)=\mathrm{OC}(G)$ ). A finite group $G$ is called OD-characterizable (resp. OC-characterizable) if $h_{\mathrm{OD}}(G)=1$ (resp. $h_{\mathrm{OC}}(G)=1$ ). Let $C=C_{p}(2)$ be a symplectic group over the binary field, for which $2^{p}-1>7$ is a Mersenne prime. The aim of this article is to prove that $h_{\mathrm{OD}}(C)=1=h_{\mathrm{OC}}(C)$.
\end{abstract}

Mathematics Subject Classification 2010: 20D05, 20D06, 20D08

Keywords: Spectrum of a group, prime graph, degree pattern, order component, symplectic group $C_{n}(q), \mathrm{OD}(\mathrm{OC})$-characterizability of a finite group

\section{Introduction}

Only finite groups will be considered. Let $G$ be a group, $\pi(G)$ the set of all prime divisors of its order and $\omega(G)$ be the spectrum of $G$, that is the set of its element orders. The prime graph $\mathrm{GK}(G)$ (or Gruenberg-Kegel graph) of $G$ is a simple graph whose vertex set is $\pi(G)$ and two distinct vertices $p$ and $q$ are joined by an edge if and only if $p q \in \omega(G)$. Let $t(G)$ be the number of connected components of $\operatorname{GK}(G)$. The vertex set of the $i$ th connected component of $\operatorname{GK}(G)$ is denoted by $\pi_{i}(G)$ for each $i=1,2, \ldots, t(G)$. In the case when $2 \in \pi(G)$, we assume that $2 \in \pi_{1}(G)$. The classification of finite simple groups with disconnected prime graph was obtained by Williams [13] and Kondratév [4]. Recall that a clique in a graph is a set of pairwise adjacent vertices. Note that for all non-abelian simple groups $S$ with disconnected prime graph, all connected components $\pi_{i}(S)$ for $2 \leqslant i \leqslant t(S)$ are cliques, for instance, see [13]. The degree $\operatorname{deg}_{G}(p)$ of a vertex $p \in \pi(G)$ in $\operatorname{GK}(G)$ is the number of edges incident on $p$. If $\pi(G)=\left\{p_{1}, p_{2}, \ldots, p_{h}\right\}$ with $p_{1}<p_{2}<\cdots<p_{h}$, then we define

$$
\mathrm{D}(G)=\left(\operatorname{deg}_{G}\left(p_{1}\right), \operatorname{deg}_{G}\left(p_{2}\right), \ldots, \operatorname{deg}_{G}\left(p_{h}\right)\right),
$$


which is called the degree pattern of $G$. Given a group $G$, denote by $h_{\mathrm{OD}}(G)$ the number of isomorphism classes of groups with the same order and degree pattern as $G$. All finite groups, in terms of the function $h_{\mathrm{OD}}(\cdot)$, are classified as follows:

Definition 1.1. A group $G$ is called $k$-fold OD-characterizable if $h_{\mathrm{OD}}(G)=k$. Usually, a 1-fold OD-characterizable group is simply called OD-characterizable.

There are scattered results in the literature showing that certain simple groups are $k$-fold OD-characterizable for $k \in\{1,2\}$. The most recent version of the list of such simple groups is presented in [8, Tables 2 and 3]. Until now, no examples of simple groups $S$ with $h_{\mathrm{OD}}(S) \geqslant 3$ were known. Therefore, we posed the following question:

Problem 1.2. Is there a non-abelian simple group $S$ with $h_{\mathrm{OD}}(S) \geqslant 3$ ?

In this article, we focus our attention on the symplectic groups $C_{p}(2) \cong S_{2 p}(2)$, where $p$ is an odd prime. Recall that $C_{2}(2)$ is not a simple group, in fact, the derived subgroup $C_{2}(2)^{\prime}$ is a simple group which is isomorphic with $A_{6} \cong L_{2}(9)$. In addition, we recall that $B_{2}(3) \cong{ }^{2} A_{4}\left(2^{2}\right), B_{n}\left(2^{m}\right) \cong C_{n}\left(2^{m}\right)$ and $B_{2}(q) \cong C_{2}(q)$ (see [2]). Previously, it was determined the values of $h_{\mathrm{OD}}(\cdot)$ for some symplectic and orthogonal groups (see $[1,6,9])$. In the table below, $\pi(n)$ is the set of all prime divisors of $n$, where $n$ is a natural number.

\begin{tabular}{llcc}
\hline$G$ & Restrictions on $G$ & $h_{\mathrm{OD}}(G)$ & Refs. \\
\hline$B_{3}(4) \cong C_{3}(4)$ & & 1 & {$[6]$} \\
$B_{2}(q) \cong C_{2}(q)$ & $\left|\pi\left(\frac{q^{2}+1}{(2, q-1)}\right)\right|=1$ & 1 & {$[1]$} \\
$B_{2^{m}}(q) \cong C_{2^{m}}(q)$ & $\left|\pi\left(\frac{q^{2}+1}{(2, q-1)}\right)\right|=1, \quad q$ is even & 1 & {$[1]$} \\
$B_{3}(5), C_{3}(5)$, & & 2 & {$[1]$} \\
$B_{n}(q), C_{n}(q)$, & $n=2^{m}>2, \quad\left|\pi\left(\frac{q^{n}+1}{2}\right)\right|=1$, & 2 & {$[1]$} \\
& $q$ is an odd prime power & & \\
$B_{p}(3), C_{p}(3)$, & $\left|\pi\left(\frac{3^{p}-1}{2}\right)\right|=1, \quad p$ is an odd prime, & 2 & {$[1,9]$} \\
\hline
\end{tabular}

Given a group $G$, the order of $G$ can be expressed as a product of some coprime natural numbers $m_{i}(G), i=1,2, \ldots, t(G)$, with $\pi\left(m_{i}(G)\right)=\pi_{i}(G)$. The numbers $m_{1}(G), m_{2}(G), \ldots, m_{t(G)}(G)$ are called the order components of $G$. We set

$$
\mathrm{OC}(G)=\left\{m_{1}(G), m_{2}(G), \ldots, m_{t(G)}(G)\right\} .
$$


In a similar manner, we define $h_{\mathrm{OC}}(G)$ as the number of isomorphism classes of finite groups with the same set $\mathrm{OC}(G)$ of order components. Again, in terms of function $h_{\mathrm{OC}}(\cdot)$, the groups $G$ are classified as follows:

Definition 1.3. A finite group $G$ is called $k$-fold OC-characterizable if $h_{\mathrm{OC}}(G)=k$. In the case when $k=1$ the group $G$ is simply called OC-characterizable.

A Mersenne prime is a prime that can be written as $2^{p}-1$ for some prime $p$. The purpose of this article is to prove the following theorem.

Main Theorem. Let $C=C_{p}(2)$ be the symplectic group over the binary field, for which $2^{p}-1>7$ is a Mersenne prime. Then $h_{\mathrm{OD}}(C)=1=h_{\mathrm{OC}}(C)$.

It is worth noting that the values of functions $h_{\mathrm{OD}}(\cdot)$ and $h_{\mathrm{OC}}(\cdot)$ may be different. For instance, suppose $M \in\left\{B_{3}(5), C_{3}(5)\right\}$. By [13], the prime graph associated with $M$ is connected and so $\operatorname{OC}(M)=\{|M|\}=\left\{2^{9} \cdot 3^{4} \cdot 5^{9} \cdot 7 \cdot 13 \cdot 31\right\}$. On the other hand, it is easy to see that the prime graph associated with a nilpotent group is always a clique, hence, we have

$$
h_{\mathrm{OC}}(M)>\nu_{\text {nil }}(|M|) \geqslant \nu_{\mathrm{a}}(|M|)=\operatorname{Par}(9)^{2} \cdot \operatorname{Par}(4)=30^{2} \times 5=4500,
$$

where $\nu_{\text {nil }}(n)$ (resp. $\left.\nu_{\mathrm{a}}(n)\right)$ signifies the number of non-isomorphic nilpotent (resp. abelian) groups of order $n$ and $\operatorname{Par}(n)$ denotes the number of partitions of $n$. However, by Theorem 1.3 in [1], we know that $h_{\mathrm{OD}}(M)=2$.

\section{Preliminaries}

If $a$ is a natural number, $r$ is an odd prime and $(r, a)=1$, then by $e(r, a)$ we denote the multiplicative order of $a$ modulo $r$, that is the minimal natural number $n$ with $a^{n} \equiv 1(\bmod r)$. If $a$ is odd, we put $e(2, a)=1$ if $a \equiv 1(\bmod 4)$, and $e(2, a)=2$ if $a \equiv-1(\bmod 4)$. The following lemma is a consequence of Zsigmondy's Theorem (see [14]).

Lemma 2.1. Let $a>1$ be an integer. Then for every natural number $n$ there exists a prime $r$ with $e(r, a)=n$ except for the cases $(n, a) \in\{(1,2),(1,3),(6,2)\}$.

A prime $r$ with $e(r, a)=n$ is called a primitive prime divisor of $a^{n}-1$. By Lemma 2.1, such a prime exists except for the cases mentioned in the lemma. We denote by $\operatorname{ppd}\left(a^{n}-1\right)$ the set of all primitive prime divisors of $a^{n}-1$. By our definition, we have $\pi(a-1)=\operatorname{ppd}(a-1)$ but for the following sole exception, namely, $2 \notin \operatorname{ppd}(a-1)$ if $e(2, a)=2$. In this case, we assume that $2 \in \operatorname{ppd}\left(a^{2}-1\right)$. 
From the definition it is easy to conclude that: Let $p>2$ be an integer. Then $\pi\left(a^{p}-1\right)=\operatorname{ppd}\left(a^{p}-1\right)$ if and only if $p$ is a prime.

In the following results, we will consider the function $\eta: \mathbb{N} \rightarrow \mathbb{N}$, which is defined as follows

$$
\eta(m)=\left\{\begin{array}{llll}
m & \text { if } & m \equiv 1 \quad(\bmod 2), \\
m / 2 & \text { if } & m \equiv 0 & (\bmod 2) .
\end{array}\right.
$$

Lemma 2.2. ([11]) Let $M$ be one of the simple groups of Lie type $B_{n}(q)$ or $C_{n}(q)$ over a field of characteristic $p$, and let $r \in \pi(M) \backslash\{p\}$ and $r \in \operatorname{ppd}\left(q^{k}-1\right)$. Then $r$ and $p$ are non-adjacent if and only if $\eta(k)>n-1$.

Lemma 2.3. ([12]) Let $M$ be one of the simple groups of Lie type $B_{n}(q)$ or $C_{n}(q)$ over a field of characteristic $p$. Let $r, s$ be odd primes with $r, s \in \pi(M) \backslash\{p\}$. Suppose that $r \in \operatorname{ppd}\left(q^{k}-1\right), s \in \operatorname{ppd}\left(q^{l}-1\right)$ and $1 \leqslant \eta(k) \leqslant \eta(l)$. Then $r$ and $s$ are non-adjacent if and only if $\eta(k)+\eta(l)>n$ and $l / k$ is not an odd natural number.

Using Lemmas 2.2 and 2.3, we conclude that the prime graphs $\operatorname{GK}\left(B_{n}(q)\right)$ and $\operatorname{GK}\left(C_{n}(q)\right)$ coincide (see also [11, Proposition 7.5]), and hence

$$
\mathrm{D}\left(B_{n}(q)\right)=\mathrm{D}\left(C_{n}(q)\right) .
$$

Corollary 2.4. Let $p>3$ be a prime and $C=C_{p}(2)$. Then $\operatorname{deg}_{C}(3)=\left|\pi_{1}(C)\right|-1$.

Proof. Recall that, by [4], we have

$$
\pi_{1}(C)=\pi\left(2\left(2^{p}+1\right) \prod_{i=1}^{p-1}\left(2^{2 i}-1\right)\right) \quad \text { and } \quad \pi_{2}(C)=\pi\left(2^{p}-1\right) .
$$

Now, it follows from Lemma 2.3 that all primitive prime divisors of $2^{p}-1$ (and so all primes in $\pi\left(2^{p}-1\right)$ ) are non-adjacent to 3 . On the other hand, by Lemmas 2.2 and 2.3, we deduce that $\operatorname{deg}_{C}(3)=\left|\pi_{1}(C)\right|-1$, as desired.

The following lemma is crucial to the study of characterizability of symplectic groups $C_{p}(2)$ by order components.

Lemma 2.5. ([3]) Let $G$ be a group whose prime graph has more than one component. If $H$ is a normal $\pi_{k}(G)$-subgroup of $G$, then $|H|-1$ is divisible by $m_{i}(G)$, for all $i \neq k$.

A group $G$ is called 2-Frobenius if there exists a normal series $1 \unlhd H \unlhd K \unlhd G$ of $G$ such that $H$ is the Frobenius kernel of $K$ and $K / H$ is the Frobenius kernel of $G / H$. 
Lemma 2.6. ([7]) Let $S$ be a simple group with disconnected prime graph $\operatorname{GK}(S)$, except $U_{4}(2)$ and $U_{5}(2)$. If $G$ is a finite group with $\mathrm{OC}(G)=\mathrm{OC}(S)$, then $G$ is neither a Frobenius group nor a 2-Frobenius group.

Lemma 2.7. ([13]) Let $G$ be a group with $t(G) \geqslant 2$. Then one of the following hold:

(1) $G$ is a Frobenius group;

(2) $G$ is a 2-Frobenius group; or

(3) $G$ has a normal series $1 \unlhd H \triangleleft K \unlhd G$ such that $H$ is a nilpotent $\pi_{1}$-group, $K / H$ is a non-abelian simple group, $G / K$ is a $\pi_{1}$-group, $|G / K|$ divides $|\operatorname{Out}(K / H)|$ and any odd order component of $G$ is equal to one of the odd order components of $K / H$.

Lemma 2.8. ([5]) The only solution of the equation $p^{m}-q^{n}=1$, where $p, q$ are primes and $m, n>1$ are integers, is $(p, q, m, n)=(3,2,2,3)$.

Given a natural number $B$ and a prime number $t$, we denote by $B_{t}$ the $t$-part of $B$, that is the largest power of $t$ dividing $B$.

Lemma 2.9. ([10]) Let $B=\left(2^{2}-1\right)\left(2^{4}-1\right) \cdots\left(2^{2 n}-1\right)$. If $t$ is a prime divisor of $B$, then $B_{t}<2^{3 n}$. Furthermore, if $t \geqslant 5$ then $B_{t}<2^{2 n}$.

\section{Proof of the main theorem}

Throughout this section, we will assume that $2^{p}-1>7$ is a Mersenne prime and $C=C_{p}(2)$. Suppose that $G$ is a group with the same order and degree pattern as $C$, that is

$$
|G|=|C|=2^{p^{2}} \prod_{i=1}^{p}\left(2^{2 i}-1\right) \text { and } \mathrm{D}(G)=\mathrm{D}(C) .
$$

Note that, according to the results summarized in [4], we have $t(C)=2$, and

$$
\pi_{1}(C)=\pi\left(2\left(2^{p}+1\right) \prod_{i=1}^{p-1}\left(2^{2 i}-1\right)\right) \quad \text { and } \quad \pi_{2}(C)=\left\{2^{p}-1\right\} .
$$

By our hypothesis, it is easy to see that

$$
\pi_{2}(G)=\pi_{2}(C)=\left\{2^{p}-1\right\} \text { and } \pi(G)=\pi(C)=\pi_{1}(C) \cup\left\{2^{p}-1\right\} .
$$

First of all, we notice that $2^{p}-1$ is the largest prime in $\pi(G)=\pi(C)$. Moreover, it follows from Corollary 2.4 that

$$
\operatorname{deg}_{G}(3)=\operatorname{deg}_{C}(3)=\left|\pi_{1}(C)\right|-1,
$$


and this forces $\pi_{1}(G)=\pi_{1}(C)$, and so $t(G)=2$. Hence, we have

$$
\mathrm{OC}(G)=\mathrm{OC}(C)=\left\{2^{p^{2}}\left(2^{p}+1\right) \prod_{i=1}^{p-1}\left(2^{2 i}-1\right), 2^{p}-1\right\},
$$

and from Lemma 2.6, the group $G$ is neither a Frobenius group nor a 2-Frobenius group. Finally, Lemma 2.7, reduces the problem to the study of the simple groups. Indeed, by Lemma 2.7, there is a normal series $1 \unlhd H \triangleleft K \unlhd G$ of $G$ such that:

(1) $H$ is a nilpotent $\pi_{1}(G)$-group, $K / H$ is a non-abelian simple group and $G / K$ is a $\pi_{1}(G)$-group. Moreover, we have $K / H \leqslant G / H \leqslant \operatorname{Aut}(K / H)$, and $t(K / H) \geqslant t(G) \geqslant 2$,

(2) $2^{p}-1$ is the only odd order component of $G$ which is equal to one of those of the quotient $K / H$,

(3) $|G / K|$ divides $|\operatorname{Out}(K / H)|$.

For odd order components of $K / H$ see $[4,13]$. Now, we will continue the proof step by step.

Step 3.1. $K / H \nsubseteq{ }^{2} A_{3}(2),{ }^{2} F_{4}(2)^{\prime},{ }^{2} A_{5}(2), E_{7}(2), E_{7}(3), A_{2}(4),{ }^{2} E_{6}(2)$ nor one of the sporadic simple groups.

Note that either the odd order components of above groups are not equal to a Mersenne prime $2^{p}-1>7$ or their orders do not divide the order of $G$.

In the following, $\mathbb{A}_{n}$ denotes the alternating group on $n$ letters.

Step 3.2. $K / H \nsubseteq \mathbb{A}_{n}$, where $n$ and $n-2$ are both prime numbers.

In this case, it follows that $n=2^{p}-1$. Now, simple computations show that

$$
\left|\mathbb{A}_{n}\right|_{2}=\left(\frac{n !}{2}\right)_{2}=2^{\left(\left[\frac{n}{2}\right]+\left[\frac{n}{2^{2}}\right]+\cdots\right)-1}=2^{2^{p}-p-2} .
$$

If $p>5$, then $2^{p}-p-2>p^{2}$ and hence the 2-part of $\left|\mathbb{A}_{n}\right|$ does not divide the 2-part of $|G|$, i.e. $2^{p^{2}}$, which is a contradiction. In the case when $p=5$, then $n=31$ and $|K / H|=(31$ ! $) / 2$, which does not divide $|G|=\left|C_{5}(2)\right|=2^{25} \cdot 3^{6} \cdot 5^{2} \cdot 7 \cdot 11 \cdot 17 \cdot 31$, which is again a contradiction.

Step 3.3. $K / H \nsubseteq \mathbb{A}_{n}$, where $n=q, q+1$, or $q+2$ ( $q$ is a prime), and one of $n$, $n-2$ is not prime.

Here, $q$ is the only odd order component of $K / H$, and so $q=2^{p}-1$. We now consider the alternating group $\mathbb{A}_{q}$ which is a subgroup of $K / H \cong \mathbb{A}_{n}$. Similar arguments as those in the previous step, on the subgroup $\mathbb{A}_{q}$ instead of $\mathbb{A}_{n}$, lead us a contradiction. 
Step 3.4. $K / H$ is isomorphic to neither ${ }^{2} E_{6}(q), q>2$, nor $E_{6}(q)$.

We deal with ${ }^{2} E_{6}(q), q>2$, the proof for $E_{6}(q)$ being quite similar. Suppose that $K / H \cong{ }^{2} E_{6}(q)$. First of all, we recall that

$$
\left|{ }^{2} E_{6}(q)\right|=\frac{1}{(3, q+1)} q^{36}\left(q^{12}-1\right)\left(q^{9}+1\right)\left(q^{8}-1\right)\left(q^{6}-1\right)\left(q^{5}+1\right)\left(q^{2}-1\right) .
$$

Considering the only odd order component of ${ }^{2} E_{6}(q)$, that is $\left(q^{6}-q^{3}+1\right) /(3, q+1)$, we must have $\left(q^{6}-q^{3}+1\right) /(3, q+1)=2^{p}-1$, which implies that $q^{9}>2^{p}$, or equivalently $q^{36}>2^{4 p}$. Let $q=r^{f}$. If $r$ is an odd prime, then from Lemma 2.9, we get

$$
q^{36}=r^{36 f}=|K / H|_{r} \leqslant|G|_{r}<2^{3 p},
$$

which is a contradiction. Therefore we may assume that $r=2$. In this case, we have

$$
\left(2^{6 f}-2^{3 f}+1\right) /\left(3,2^{f}+1\right)=2^{p}-1 .
$$

Now, if $\left(3,2^{f}+1\right)=1$, then we obtain $2^{3 f}\left(2^{3 f}-1\right)=2\left(2^{p-1}-1\right)$, from which we deduce that $3 f=1$, a contradiction. In the case where $\left(3,2^{f}+1\right)=3$, an easy calculation shows that

$$
2^{3 f}\left(2^{3 f}-1\right)=2^{2}\left(3 \cdot 2^{p-2}-1\right),
$$

and so $3 f=2$, which is again a contradiction.

Step 3.5. $K / H \nsubseteq F_{4}(q)$, where $q$ is an odd prime power.

We remark that $q^{4}-q^{2}+1$ is the only odd order component of $F_{4}(q)$, and clearly this forces $q^{4}-q^{2}+1=2^{p}-1$. Then $q^{2}\left(q^{2}-1\right)=2\left(2^{p-1}-1\right)$, which shows that $2\left(2^{p-1}-1\right)$ is divisible by 4 , a contradiction.

Step 3.6. $K / H \varlimsup^{2} B_{2}(q)$, where $q=2^{2 m+1}>2$.

Recall that $\left|{ }^{2} B_{2}(q)\right|=q^{2}\left(q^{2}+1\right)(q-1)$ and the odd order components of ${ }^{2} B_{2}(q)$ are:

$$
q-1, \quad q-\sqrt{2 q}+1, \quad q+\sqrt{2 q}+1
$$

If $q-1=2^{p}-1$, then $q=2^{p}$. Now, we consider the primitive prime divisor $r \in \operatorname{ppd}\left(2^{4 p}-1\right)$. Clearly $r \in \pi\left(2^{2 p}+1\right)$, and so $r \in \pi\left({ }^{2} B_{2}(q)\right) \subseteq \pi(G)$. This is a contradiction.

In the case when

$$
q-\sqrt{2 q}+1=2^{p}-1 \quad\left(\text { resp. } q+\sqrt{2 q}+1=2^{p}-1\right)
$$


by simple computations we obtain

$$
2^{m+1}\left(2^{m}-1\right)=2\left(2^{p-1}-1\right) \quad\left(\text { resp. } 2^{m+1}\left(2^{m}+1\right)=2\left(2^{p-1}-1\right)\right),
$$

a contradiction.

Step 3.7. $K / H \nsubseteq E_{8}(q)$, where $q \equiv 2,3(\bmod 5)$.

The odd order components of $E_{8}(q)$ in this case are

$$
q^{8}-q^{4}+1, \quad \frac{q^{10}+q^{5}+1}{q^{2}+q+1}, \quad \frac{q^{10}-q^{5}+1}{q^{2}-q+1} .
$$

If $q^{8}-q^{4}+1=2^{p}-1$, then we obtain $q^{4}(q-1)(q+1)\left(q^{2}+1\right)=2\left(2^{p-1}-1\right)$. However, the left hand side is divisible by 16, while the right hand side is not divisible by 4 , which is impossible.

If $\left(q^{10}+q^{5}+1\right) /\left(q^{2}+q+1\right)=2^{p}-1$, then after subtracting 1 from both sides of this equation and some simple computations, we obtain

$$
q(q-1)(q+1)\left(q^{2}+1\right)\left(q^{3}-q^{2}+1\right)=2\left(2^{p-1}-1\right) .
$$

Now, if $q$ is odd, then the left hand side is divisible by 16, a contradiction. Moreover, if $q$ is even, then it follows that $q=2$, and if this is substituted in above equation we get $76=2^{p-1}$, a contradiction.

The case $\left(q^{10}-q^{5}+1\right) /\left(q^{2}-q+1\right)=2^{p}-1$ is quite similar to the previous case and it is omitted.

Step 3.8. $K / H \nsubseteq E_{8}(q)$, where $q \equiv 0,1,4(\bmod 5)$.

The odd order components of $E_{8}(q)$ in this case are

$$
\frac{q^{10}+1}{q^{2}+1}, \quad q^{8}-q^{4}+1, \quad \frac{q^{10}+q^{5}+1}{q^{2}+q+1}, \quad \frac{q^{10}-q^{5}+1}{q^{2}-q+1} .
$$

Consider the first case. Let $\left(q^{10}+1\right) /\left(q^{2}+1\right)=2^{p}-1$. Subtracting 1 from both sides of this equality, we get

$$
q^{2}\left(q^{2}-1\right)\left(q^{4}+1\right)=2\left(2^{p-1}-1\right),
$$

which implies $2\left(2^{p-1}-1\right)$ is divisible by 4 , a contradiction.

Similarly, if $q^{8}-q^{4}+1=2^{p}-1$, we obtain $q^{4}(q-1)(q+1)\left(q^{2}+1\right)=2\left(2^{p-1}-1\right)$, which shows that $2\left(2^{p-1}-1\right)$ is divisible by 16 , a contradiction. Similar arguments work if $\left(q^{10}+q^{5}+1\right) /\left(q^{2}+q+1\right)=2^{p}-1$ or $\left(q^{10}-q^{5}+1\right) /\left(q^{2}-q+1\right)=2^{p}-1$, and we omit the details.

Step 3.9. $K / H \oiint^{2} F_{4}(q)$, where $q=2^{2 m+1}>2$. 
The odd order components of ${ }^{2} F_{4}(q)$ are:

$$
q^{2}+\sqrt{2 q^{3}}+q+\sqrt{2 q}+1 \text { and } q^{2}-\sqrt{2 q^{3}}+q-\sqrt{2 q}+1 .
$$

Therefore, we have

$$
q^{2}+\sqrt{2 q^{3}}+q+\sqrt{2 q}+1=2^{p}-1 \text { or } q^{2}-\sqrt{2 q^{3}}+q-\sqrt{2 q}+1=2^{p}-1 .
$$

However, if $2^{2 m+1}$ is substituted in these equations we obtain

$$
2^{m+1}\left(2^{3 m+1} \pm 2^{2 m+1}+2^{m} \pm 1\right)=2\left(2^{p-1}-1\right),
$$

which is a contradiction.

Step 3.10. $K / H \nsubseteq F_{4}(q)$, where $q=2^{m}$.

The odd order components of $F_{4}(q)$ are $q^{4}+1$ and $q^{4}-q^{2}+1$, hence $q^{4}+1=2^{p}-1$ or $q^{4}-q^{2}+1=2^{p}-1$. Now, it is easy to see that in both cases, $2^{2 m}$ divides $2\left(2^{p-1}-1\right)$, a contradiction.

Step 3.11. $K / H \not{ }^{2} G_{2}(q)$, where $q=3^{2 m+1}>3$.

The odd order components of ${ }^{2} G_{2}(q)$ are $q+\sqrt{3 q}+1$ and $q-\sqrt{3 q}+1$. If $q-\sqrt{3 q}+1=2^{p}-1$, then $q^{3}>2^{3 p}$, while Lemma 2.9 shows that $q^{3}<2^{3 p}$, which is a contradiction. If $q+\sqrt{3 q}+1=2^{p}-1$, then

$$
2^{p}-2=2\left(2^{(p-1) / 2}-1\right)\left(2^{(p-1) / 2}+1\right)=3^{m+1}\left(3^{m}+1\right) .
$$

First of all, we recall that $\left(2^{(p-1) / 2}-1,2^{(p-1) / 2}+1\right)=1$. Now we consider two cases separately:

(i) If $3^{m+1}$ divides $2^{(p-1) / 2}-1$, then

$$
3^{m}+1<3^{m+1} \leqslant 2^{(p-1) / 2}-1<2^{(p-1) / 2}+1 .
$$

Hence, we obtain

$$
3^{m+1}\left(3^{m}+1\right)<2\left(2^{(p-1) / 2}-1\right)\left(2^{(p-1) / 2}+1\right),
$$

a contradiction.

(ii) If $3^{m+1}$ divides $2^{(p-1) / 2}+1$, then $2^{(p-1) / 2}+1=k \cdot 3^{m+1}$ where $k$ is a natural number. Now, from Eq.( 1$)$, it follows that

$$
2 k\left(2^{(p-1) / 2}-1\right)=3^{m}+1,
$$

and consequently $3^{m} \geqslant 2^{(p+1) / 2}-1$. Therefore we have

$$
2^{(p+1) / 2}-1 \leqslant 3^{m}<3^{m+1} \leqslant 2^{(p-1) / 2}+1,
$$

a contradiction. 
Step 3.12. $K / H \not G_{2}(q)$, where $q=3^{m}$.

Recall that the odd order components of $G_{2}(q)$ are $q^{2}-q+1$ and $q^{2}+q+1$. If $q^{2}-q+1=2^{p}-1$ then $q^{6}>2^{3 p}$, while one can follow from Lemma 2.9 that $q^{6}<2^{3 p}$, which is a contradiction. If $q^{2}+q+1=2^{p}-1$, then $q(q+1) \equiv 2(\bmod 4)$, which forces $m$ is even. But then, it is obvious that $2^{p}-2=q(q+1) \equiv 2(\bmod 8)$, a contradiction.

Step 3.13. $K / H \nexists^{2} D_{r}(3)$, where $r=2^{m}+1$ is a prime number and $m \geqslant 1$.

Recall that

$$
\left|{ }^{2} D_{r}(3)\right|=\frac{1}{\left(4,3^{r}+1\right)} 3^{r(r-1)}\left(3^{r}+1\right) \prod_{i=1}^{r-1}\left(3^{2 i}-1\right),
$$

and the odd order components of ${ }^{2} D_{r}(3)$ are

$$
\left(3^{r-1}+1\right) / 2 \text { and }\left(3^{r}+1\right) / 4 .
$$

In the case when $\left(3^{r-1}+1\right) / 2=2^{p}-1$, adding 1 to both sides of this equality, we obtain

$$
3\left(3^{r-2}+1\right)=2^{p+1},
$$

which is a contradiction. If $\left(3^{r}+1\right) / 4=2^{p}-1$, then $r \geqslant 5$ because $p \geqslant 5$. Moreover, on the one hand, from last equation we obtain $3^{r}=2^{p+2}-5>2^{p+1}$, which implies that

$$
3^{r(r-1)}>2^{(p+1)(r-1)}>2^{4(p+1)} .
$$

On the other hand, it follows from Lemma 2.9 that

$$
3^{r(r-1)}=|K / H|_{3} \leqslant|G|_{3}<2^{3 p},
$$

which is a contradiction.

Step 3.14. $K / H \not B_{n}(q)$, where $n=2^{m} \geqslant 4$ and $q=r^{f}$ is an odd prime power.

Note that

$$
\left|B_{n}(q)\right|=\frac{1}{(2, q-1)} q^{n^{2}} \prod_{i=1}^{n}\left(q^{2 i}-1\right),
$$

and the only odd order component of $B_{n}(q)$ is $\left(q^{n}+1\right) / 2$. If $\left(q^{n}+1\right) / 2=2^{p}-1$, then $q^{n}=2^{p+1}-3>2^{p}$ and clearly $q$ is not divisible by 2 and 3 . Since $p \geqslant 5$ and $n \geqslant 4$, it is easy to see that

$$
q^{n^{2}}>q^{3 n}>2^{3 p}>2^{2 p}
$$

On the other hand, by Lemma 2.9 , we obtain

$$
q^{n^{2}}=|K / H|_{r} \leqslant|G|_{r}<2^{2 p}
$$


which is a contradiction.

Step 3.15. $K / H \nsubseteq B_{r}(3)$.

The only odd order component of $B_{r}(3)$ is $\left(3^{r}-1\right) / 2$. If $\left(3^{r}-1\right) / 2=2^{p}-1$, then $2^{p+1}-3^{r}=1$. However, this equation has no solution by Lemma 2.8 , which is impossible.

Step 3.16. $K / H \nsubseteq{ }^{3} D_{4}(q)$.

We recall that $q^{4}-q^{2}+1$ is the only odd order component of ${ }^{3} D_{4}(q)$, and so $q^{4}-q^{2}+1=2^{p}-1$. But then, $q^{2}\left(q^{2}-1\right)=2\left(2^{p-1}-1\right)$, which shows that $2\left(2^{p-1}-1\right)$ is divisible by 4 , a contradiction.

Step 3.17. $K / H \nsubseteq G_{2}(q)$, where $2<q \equiv \pm 1(\bmod 3)$.

In this case, the odd order components of $G_{2}(q)$ are $q^{2}+q+1$ and $q^{2}-q+1$. Let $q=r^{f}$. If $q^{2}+q+1=2^{p}-1$, then $q(q+1)=2\left(2^{p-1}-1\right)$, which shows that $q>2$ is not a power of 2 . Moreover, since $q-1 \geqslant 2$, we obtain

$$
q^{3}-1=(q-1)\left(q^{2}+q+1\right) \geqslant 2\left(2^{p}-1\right),
$$

and so $q^{3} \geqslant 2^{p+1}-1>2^{p}$, which yields that $q^{6}>2^{2 p}$. However, since

$$
\left|G_{2}(q)\right|=q^{6}\left(q^{2}-1\right)\left(q^{6}-1\right),
$$

from Lemma 2.9, we conclude that

$$
q^{6}=|K / H|_{r} \leqslant|G|_{r}<2^{2 p}
$$

which is a contradiction.

The case when $q^{2}-q+1=2^{p}-1$ is similar and left to the reader.

Step 3.18. $K / H \oiint^{2} D_{n}(3)$, where $n=2^{m}+1$ which is not a prime and $m \geqslant 2$.

The odd order component of ${ }^{2} D_{n}(3)$ is $\left(3^{n-1}+1\right) / 2$. If $\left(3^{n-1}+1\right) / 2=2^{p}-1$, then $2^{p+1}=3\left(3^{n-2}+1\right)$, a contradiction.

Step 3.19. $K / H \nsubseteq{ }^{2} D_{r}(3)$, where $r \geqslant 5$ is a prime and $r \neq 2^{m}+1$.

Here, we have

$$
\left|{ }^{2} D_{r}(3)\right|=\frac{1}{\left(4,3^{r}+1\right)} 3^{r(r-1)}\left(3^{r}+1\right) \prod_{i=1}^{r-1}\left(3^{2 i}-1\right) .
$$


The only odd order component of ${ }^{2} D_{r}(3)$ is $\left(3^{r}+1\right) / 4$, and so $\left(3^{r}+1\right) / 4=2^{p}-1$. An easy computation shows that $3^{r}=2^{p+2}-5>2^{p+1}$. Moreover, we note that $r-1 \geqslant 4$, and so

$$
3^{r(r-1)} \geqslant 3^{4 r}>2^{4(p+1)} .
$$

On the other hand, by Lemma 2.9 , we obtain

$$
3^{r(r-1)}=|K / H|_{3} \leqslant|G|_{3}<2^{3 p},
$$

which is a contradiction.

Step 3.20. $K / H \not^{2} D_{n}(2)$, where $n=2^{m}+1, m \geqslant 2$.

The only odd order component of ${ }^{2} D_{n}(2)$ is $2^{n-1}+1$. Therefore, we obtain $2^{n-1}+1=2^{p}-1$, which is impossible.

Step 3.21. $K / H \not^{2} D_{n}(q)$, where $n=2^{m} \geqslant 4$ and $q=r^{f}$.

Recall that

$$
\left.\right|^{2} D_{n}(q) \mid=\frac{1}{\left(4, q^{n}+1\right)} q^{n(n-1)}\left(q^{n}+1\right) \prod_{i=1}^{n-1}\left(q^{2 i}-1\right),
$$

and the only odd order component of ${ }^{2} D_{n}(q)$ is $\left(q^{n}+1\right) /(2, q+1)$. Therefore, $\left(q^{n}+1\right) /(2, q+1)=2^{p}-1$. Assume first that $(2, q+1)=1$. In this case, we obtain $q^{n}=2\left(2^{p-1}-1\right)$, a contradiction. Assume next that $(2, q+1)=2$. Again, using simple calculations we obtain $q^{n}=2^{p+1}-3>2^{p}$ and so $q$ cannot be a power of 2 . Moreover, since $n-1 \geqslant 3, q^{n(n-1)} \geqslant q^{3 n}>2^{3 p}$. Now, Lemma 2.9 shows that

$$
q^{n(n-1)}=|K / H|_{r} \leqslant|G|_{r}<2^{3 p},
$$

which is a contradiction.

Step 3.22. $K / H \nsubseteq D_{r+1}(q)$, where $q=2,3$.

Since, the only odd order component of $D_{r+1}(q)$ is $\left(q^{r}-1\right) /(2, q-1)$, we have $\left(q^{r}-1\right) /(2, q-1)=2^{p}-1$. If $(2, q-1)=1$, then $r=p$ and $q=2$, and we have

$$
|K / H|=\left|D_{p+1}(2)\right|=\frac{1}{\left(4,2^{p+1}-1\right)} 2^{p(p+1)}\left(2^{p+1}-1\right) \prod_{i=1}^{p}\left(2^{2 i}-1\right),
$$

this shows that $|K / H|_{2}=2^{p(p+1)} /\left(4,2^{p+1}-1\right)$ does not divide $|G|_{2}=2^{p^{2}}$, which is a contradiction. In the case when $(2, q-1)=2$, we have the equation $2^{p+1}-3^{r}=1$, which has no solution for $p \geqslant 5$, by Lemma 2.8. This is again a contradiction.

Step 3.23. $K / H \nsubseteq D_{r}(q)$, where $q=2,3,5$ and $r \geqslant 5$. 
We recall that the only odd order component of $D_{r}(q)$ is $\left(q^{r}-1\right) /(q-1)$. We distinguish three cases separately.

(i) $q=2$. In this case, we have $2^{r}-1=2^{p}-1$, and so $r=p$ and

$$
|K / H|=\left|D_{p}(2)\right|=2^{p(p-1)}\left(2^{p}-1\right) \prod_{i=1}^{p-1}\left(2^{2 i}-1\right) .
$$

Note that $\left|\operatorname{Out}\left(D_{p}(2)\right)\right|=2$ and $D_{p}(2) \leqslant G / H \leqslant \operatorname{Aut}\left(D_{p}(2)\right)$. Now, considering the order of groups, we get $|H|=2^{\alpha}\left(2^{p}+1\right)$ where $p-1 \leqslant \alpha \leqslant p$. Let $r \in \operatorname{ppd}\left(2^{2 p}-1\right)$ and $Q \in \operatorname{Syl}_{r}(H)$. Clearly $r \in \pi\left(2^{p}+1\right), Q$ is a normal $\pi_{1}(G)$-subgroup of $G$ and $|Q|$ divides $2^{p}+1$. Now, from Lemma 2.5, it follows that $|Q|-1$ is divisible by $m_{2}(G)=2^{p}-1$, and so $|Q|-1 \geqslant 2^{p}-1$ or equivalently $|Q| \geqslant 2^{p}$. This forces $|Q|=2^{p}+1$. But then $m_{2}(G)=2^{p}-1$ does not divide the value $|Q|-1=2^{p}$, which is a contradiction.

(ii) $q=3$. In this case, from the equality $\left(3^{r}-1\right) / 2=2^{p}-1$, we deduce that $2^{p+1}-3^{r}=1$. However, this equation has no solution when $p \geqslant 5$ by Lemma 2.8, a contradiction.

(iii) $q=5$. Here $\left(5^{r}-1\right) / 4=2^{p}-1$, and so $5^{r}=2^{p+2}-3>2^{p+1}$. As before, since $r-1 \geqslant 4$, we obtain $5^{r(r-1)}>5^{4 r}>2^{4(p+1)}$. On the other hand, by Lemma 2.9, we have

$$
5^{r(r-1)}=|K / H|_{5} \leqslant|G|_{5}<2^{2 p},
$$

which is a contradiction.

Step 3.24. $K / H \not C_{r}(3)$.

The only odd order component of $C_{r}(3)$ is $\left(3^{r}-1\right) / 2$. Thus, if $\left(3^{r}-1\right) / 2=2^{p}-1$, then $2^{p+1}-3^{r}=1$. However, this equation has no solution by Lemma 2.8 , which is impossible.

Step 3.25. $K / H \nsubseteq C_{n}(q)$, where $n=2^{m} \geqslant 2$.

Note that

$$
\left|C_{n}(q)\right|=\frac{1}{(2, q-1)} q^{n^{2}} \prod_{i=1}^{n}\left(q^{2 i}-1\right),
$$

and the only odd order component of $C_{n}(q)$ is $\left(q^{n}+1\right) /(2, q-1)$. Therefore, $\left(q^{n}+1\right) /(2, q-1)=2^{p}-1$. If $(2, q-1)=1$, then $q^{n}=2\left(2^{p-1}-1\right)$, which yields that $q=p=2$ and $n=1$, a contradiction. If $(2, q-1)=2$, then $q^{n}=2^{p+1}-3>2^{p}$, which implies that $q$ is not a power of 2 and 3. Let $q=r^{f}$. When $n \geqslant 4$, it is easy to see that

$$
q^{n^{2}}>q^{3 n}>2^{3 p}>2^{2(p+1)} .
$$


But, from Lemma 2.9, we obtain

$$
q^{n^{2}}=|K / H|_{r} \leqslant|G|_{r}<2^{2 p}
$$

a contradiction. Assume now that $n=2$. In this case, we have $q^{2}=2^{p+1}-3$, or equivalently

$$
(q-1)(q+1)=2^{2}\left(2^{p-1}-1\right) .
$$

However, the left hand side is divisible by 8 , while the right hand side is divisible by 4 , a contradiction.

Step 3.26. $K / H \not A_{1}(q)$, where $q=2^{m}>2$.

The odd order components of $A_{1}(q)$ are $q+1$ and $q-1$. If $q+1=2^{p}-1$, then $q=2\left(2^{p-1}-1\right)$, a contradiction. If $q-1=2^{p}-1$, then $q=2^{p}$. Moreover, since $A_{1}(q) \leqslant G / H \leqslant \operatorname{Aut}\left(A_{1}(q)\right)$, it is easy to see that the order of $H$ is divisible by $\left(2^{2}-1\right)\left(2^{4}-1\right) \cdots\left(2^{2(p-1)}-1\right)$. Let $r \in \operatorname{ppd}\left(2^{2(p-1)}-1\right)$ and $Q \in \operatorname{Syl}_{r}(H)$. Clearly $Q$ is a normal $\pi_{1}(G)$-subgroup of $G$ and $|Q|$ divides $2^{p-1}+1$. On the other hand, from Lemma $2.5,|Q|-1$ is divisible by $2^{p}-1$ which implies that $|Q| \geqslant 2^{p}$. This is a contradiction.

Step 3.27. $K / H \nsubseteq A_{1}(q)$, where $3 \leqslant q \equiv \pm 1(\bmod 4)$ and $q=r^{f}$.

Assume first that $3 \leqslant q \equiv 1(\bmod 4)$. In this case, the odd order components of $A_{1}(q)$ are $(q+1) / 2$ and $q$. If $(q+1) / 2=2^{p}-1$, then $r^{f}=q=2^{p+1}-3$. First of all, we claim that $f$ is an odd number. Otherwise, we have

$$
\left(r^{f / 2}-1\right)\left(r^{f / 2}+1\right)=2^{2}\left(2^{p-1}-1\right) .
$$

But then, the left hand side is divisible by 8 , while the right hand side is divisible by 4 , which is a contradiction. Furthermore, by easy computations we observe that

$$
\left|A_{1}(q)\right|=\frac{1}{2} q\left(q^{2}-1\right)=2^{2}\left(2^{p+1}-3\right)\left(2^{p-1}-1\right)\left(2^{p}-1\right) .
$$

On the other hand, we have $|G / K| \cdot|H|=|G| /\left|A_{1}(q)\right|$, from which we deduce that

$$
|G / K|_{2} \cdot|H|_{2}=\frac{|G|_{2}}{\left|A_{1}(q)\right|_{2}}=2^{p^{2}-2} .
$$

But since $|G / K|$ divides $\left|\operatorname{Out}\left(A_{1}(q)\right)\right|=2 f$ and $f$ is odd, $|G / K|_{2}$ is at most 2 . Hence, if $S_{2} \in \operatorname{Syl}_{2}(H)$, then $\left|S_{2}\right|=2^{p^{2}-2}$ or $\left|S_{2}\right|=2^{p^{2}-3}$. We notice that $S_{2}$ is a normal subgroup of $G$, because $H$ is nilpotent. Now, it follows from Lemma 2.5 that $2^{p}-1$ divides $2^{p^{2}-2}-1$ or $2^{p^{2}-3}-1$, which is a contradiction. If $q=2^{p}-1$, we get a contradiction by Lemma 2.8 .

Assume next that $3 \leqslant q \equiv-1(\bmod 4)$. In this case, the odd order components of $A_{1}(q)$ are $(q-1) / 2$ and $q$. If $(q-1) / 2=2^{p}-1$, then $2^{p+1}-r^{f}=1$. Noting 
Lemma 2.8, we deduce that $f=1$, and hence $r=2^{p+1}-1$ is a Mersenne prime, which is a contradiction because $p+1$ is not a prime.

The case when $q=2^{p}-1$ is similar to the previous paragraph.

Step 3.28. $K / H \nsubseteq A_{r}(q)$, where $(q-1) \mid(r+1)$.

Recall that

$$
|K / H|=\left|A_{r}(q)\right|=\frac{1}{(r+1, q-1)} q^{r(r+1) / 2} \prod_{i=2}^{r+1}\left(q^{i}-1\right) .
$$

The only odd order component of $A_{r}(q)$ is $\left(q^{r}-1\right) /(q-1)$, and so

$$
\left(q^{r}-1\right) /(q-1)=2^{p}-1 .
$$

As a simple observation we see that $q^{r}-1 \geqslant\left(q^{r}-1\right) /(q-1)=2^{p}-1$ and so $q^{r} \geqslant 2^{p}$. Let $q=t^{f}$, where $t$ is a prime number and $f$ is a natural number.

(i) Suppose first that $r \geqslant 7$. Then $q^{r(r+1) / 2}>q^{3(r+1)} \geqslant 2^{3} q^{3 r} \geqslant 2^{3(p+1)}$. Now, if $t$ is an odd prime, then by Lemma 2.9 we obtain

$$
q^{r(r+1) / 2}=|K / H|_{t} \leqslant|G|_{t}<2^{3 p},
$$

which is a contradiction. Therefore, we may assume that $t=2$. In this case, we have

$$
\left(2^{f r}-1\right) /\left(2^{f}-1\right)=2^{p}-1,
$$

from which one can deduce that $f=1$ and $r=p$. Thus

$$
|G / K| \cdot|H|=\frac{2^{p^{2}} \prod_{i=1}^{p}\left(2^{2 i}-1\right)}{2^{\frac{p(p+1)}{2}} \prod_{i=2}^{p+1}\left(2^{i}-1\right)} .
$$

Since $|G / K|$ divides $|\operatorname{Out}(K / H)|=\left|\operatorname{Out}\left(A_{p}(2)\right)\right|=2$, we conclude that $|H|$ is divisible by $2^{p}+1$. Let $s \in \operatorname{ppd}\left(2^{2 p}-1\right) \subseteq \pi\left(2^{p}+1\right)$ and $Q \in \operatorname{Syl}_{s}(H)$. Clearly $\mid Q \| 2^{p}+1$. Since $H$ is a normal $\pi_{1}(G)$-subgroup of $G$ which is nilpotent, $Q$ is also a normal $\pi_{1}(G)$-subgroup of $G$. Now, by Lemma 2.5, $m_{2}(G)=2^{p}-1$ divides $|Q|-1$, and so $|Q| \geqslant 2^{p}$. But, this forces $|Q|=2^{p}+1$. However, this contradicts the fact that $m_{2}(G)|| Q \mid-1$.

(ii) Suppose next that $r=5$. If $q$ is even, then from $\left(q^{5}-1\right) /(q-1)=2^{p}-1$, we obtain $q\left(q^{3}+q^{2}+q+1\right)=2\left(2^{p-1}-1\right)$, which implies that $q=2$ and $r=p=5$. Therefore, by easy calculations we see that

$$
|G / K| \cdot|H|=\frac{2^{10} \prod_{i=1}^{5}\left(2^{i}+1\right)}{2^{6}-1},
$$

which is not a natural number, a contradiction. If $q$ is odd, then we get

$$
q(q+1)\left(q^{2}+1\right)=q^{4}+q^{3}+q^{2}+q=2^{p}-2,
$$


however $q(q+1)\left(q^{2}+1\right) \equiv 0(\bmod 4)$, while $2^{p}-2 \equiv 2(\bmod 4)$, a contradiction.

(iii) Finally suppose that $r=3$. Then $q(q+1)=2\left(2^{p-1}-1\right)$. First of all, we note that $q$ is not even, otherwise $p=3$, which is impossible. In addition, we have

$$
q(q+1)=2\left(2^{(p-1) / 2}-1\right)\left(2^{(p-1) / 2}+1\right) .
$$

Now we consider two cases separately:

(a) If $q$ divides $2^{(p-1) / 2}-1$, then

$$
q \leqslant 2^{(p-1) / 2}-1, \quad q+1<2^{(p-1) / 2}+1 .
$$

Hence, we obtain

$$
q(q+1)<2\left(2^{(p-1) / 2}-1\right)\left(2^{(p-1) / 2}+1\right),
$$

a contradiction.

(b) If $q$ divides $2^{(p-1) / 2}+1$, then $2^{(p-1) / 2}+1=k q$ for some natural number $k$. Now from Eq. (2), it follows that

$$
2 k\left(2^{(p-1) / 2}-1\right)=q+1 .
$$

If $k=1$, then $p=q=5$. Hence $13 \in \pi(K / H)=\pi\left(A_{3}(5)\right)$, however $13 \notin \pi(G)=\pi\left(C_{5}(2)\right)$, a contradiction. Thus, $k \geqslant 2$ and we obtain

$$
2\left(2^{(p+1) / 2}-2\right)-1 \leqslant q<q+1 \leqslant k q=2^{(p-1) / 2}+1,
$$

which is a contradiction.

Step 3.29. $K / H \nsubseteq A_{r-1}(q)$, where $(r, q) \neq(3,2),(3,4)$.

Again, we recall that

$$
|K / H|=\left|A_{r-1}(q)\right|=\frac{1}{(r, q-1)} q^{r(r-1) / 2} \prod_{i=2}^{r}\left(q^{i}-1\right),
$$

and the only odd order component of $A_{r-1}(q)$ is $\left(q^{r}-1\right) /(q-1)(r, q-1)$. Hence, we must have

$$
\left(q^{r}-1\right) /(q-1)(r, q-1)=2^{p}-1
$$

which implies that

$$
q^{r}-1 \geqslant\left(q^{r}-1\right) /(q-1)(r, q-1)=2^{p}-1,
$$

or equivalently $q^{r} \geqslant 2^{p}$. Let $q=t^{f}$, where $t$ is a prime and $f$ is a natural number. In what follows, we consider several cases separately. 
(i) $r \geqslant 7$. In this case, we obtain

$$
q^{r(r-1) / 2} \geqslant q^{3 r} \geqslant 2^{3 p}
$$

and Lemma 2.9 implies that $t=2$. Now, Lemma 2.1 shows that $q=2$ and $r=p$, and hence we obtain

$$
|G / K| \cdot|H|=\frac{2^{p^{2}} \prod_{i=1}^{p}\left(2^{2 i}-1\right)}{2^{\left(\begin{array}{c}
p \\
2
\end{array}\right)} \prod_{i=2}^{p}\left(2^{i}-1\right)}=2^{\frac{p(p+1)}{2}} \prod_{i=1}^{p}\left(2^{i}+1\right) .
$$

On the other hand, $|G / K|$ divides $|\operatorname{Out}(K / H)|=2$. From this we deduce that $|H|$ is divisible by $2^{p}+1$. Let $s \in \operatorname{ppd}\left(2^{2 p}-1\right) \subseteq \pi\left(2^{p}+1\right)$ and $Q \in \operatorname{Syl}_{s}(H)$. Evidently $Q$ is a normal subgroup of $G$ and $|Q|$ divides $2^{p}+1$. Now, it follows from Lemma 2.5 that $m_{2}(G)=2^{p}-1|| Q \mid-1$, which is impossible.

(ii) $r=5$. Assume first that $(5, q-1)=1$. In this case, we have

$$
\frac{q^{5}-1}{q-1}=q^{4}+q^{3}+q^{2}+q+1=2^{p}-1,
$$

or equivalently

$$
q(q+1)\left(q^{2}+1\right)=2\left(2^{p-1}-1\right)
$$

If $q$ is even, then we conclude that $q=2$ and $r=p=5$, and the proof is quite similar as $(i)$. If $q$ is odd, then the left-hand side of Eq.( 3 ) is congruent to $0(\bmod 4)$, while the right-hand side of Eq.( 3$)$ is congruent to $2(\bmod 4)$, a contradiction.

Assume next that $(5, q-1)=5$. In this case, we have

$$
q^{4}+q^{3}+q^{2}+q+1=5\left(2^{p}-1\right)
$$

or equivalently

$$
(q-1)\left(q^{3}+2 q^{2}+3 q+4\right)=10\left(2^{p-1}-1\right) .
$$

In the case when $q$ is even, one can easily deduce that $q=2$, and so $13=5\left(2^{p-1}-1\right)$, a contradiction. Moreover, if $q$ is odd, then from the equality $q(q+1)\left(q^{2}+1\right)=5 \cdot 2^{p}-6$ it is easily seen that the left-hand side of this equation is congruent to $0(\bmod 4)$, while the right-hand side is congruent to $2(\bmod 4)$, a contradiction.

(iii) $r=3$. In this case, we have $\left(q^{3}-1\right) /(q-1)(3, q-1)=2^{p}-1$. First of all, if $q$ is even, then we obtain $p=3$, which is not the case. Thus, we can assume that $q$ is odd. 
If $(3, q-1)=1$, then

$$
q(q+1)=2\left(2^{(p-1) / 2}-1\right)\left(2^{(p-1) / 2}+1\right) .
$$

If $q$ divides $2^{(p-1) / 2}-1$, then

$$
q \leqslant 2^{(p-1) / 2}-1, \quad q+1<2^{(p-1) / 2}+1 .
$$

Hence, we obtain

$$
q(q+1)<2\left(2^{(p-1) / 2}-1\right)\left(2^{(p-1) / 2}+1\right)
$$

a contradiction. If $q$ divides $2^{(p-1) / 2}+1$, then $2^{(p-1) / 2}+1=k q$. Now, from Eq.( 4), it follows that

$$
2 k\left(2^{(p-1) / 2}-1\right)=q+1 .
$$

When $k=1$, we conclude that $p=5$ and $q=5$. But then, we have

$$
|K / H|=\left|A_{2}(5)\right|=2^{5} \cdot 3 \cdot 5^{3} \cdot 31,
$$

while $|G|=\left|C_{5}(2)\right|=2^{25} \cdot 3^{6} \cdot 5^{2} \cdot 7 \cdot 11 \cdot 17 \cdot 31$; this is a contradiction because $|K / H|_{5}>|G|_{5}$. If $k \geqslant 2$, then $q \geqslant 2\left(2^{(p+1) / 2}-2\right)-1$. Therefore, we have

$$
2\left(2^{(p+1) / 2}-2\right)-1 \leqslant q<q+1 \leqslant 2^{(p-1) / 2}+1,
$$

a contradiction.

If $(3, q-1)=3$, then $q(q+1)=2^{2}\left(3 \cdot 2^{p-2}-1\right)$, which implies that $(q+1)_{2}=4$ and so $(q-1)_{2}=2$. Moreover, under these conditions, one can easily deduce that $f$ is odd, otherwise $8 \mid q-1$ where

$$
q-1=t^{f}-1=\left(t^{f / 2}-1\right)\left(t^{f / 2}+1\right),
$$

which is a contradiction. Thus, we have $\left|A_{2}(q)\right|_{2}=2^{4}$, while

$$
|G / K|_{2} \cdot|H|_{2}=\frac{|G|_{2}}{\left|A_{2}(q)\right|_{2}}=2^{p^{2}-4}
$$

Since $|G / K|$ divides $2 f(3, q-1)$ and $f$ is odd, $|G / K|_{2} \leqslant 2$. Therefore a Sylow 2-subgroup of $H$ has order either $2^{p^{2}-4}$ or $2^{p^{2}-5}$. Applying Lemma 2.5 we deduce that $2^{p}-1 \mid 2^{p^{2}-4}-1$ or $2^{p}-1 \mid 2^{p^{2}-5}-1$. Now, one can easily check that the second divisibility is possible only for $p=5$. But then, we get $q(q+1)=2^{2} \cdot 23$, which is a contradiction.

Step 3.30. $K / H \not{ }^{2} A_{r}(q)$, where $(q+1) \mid(r+1)$ and $(r, q) \neq(3,3),(5,2)$. 
In this case, we have

$$
|K / H|=\left|{ }^{2} A_{r}(q)\right|=\frac{1}{(r+1, q+1)} q^{r(r+1) / 2} \prod_{i=2}^{r+1}\left(q^{i}-(-1)^{i}\right),
$$

and the only odd order component of ${ }^{2} A_{r}(q)$ is $\left(q^{r}+1\right) /(q+1)$. Therefore, we get

$$
\left(q^{r}+1\right) /(q+1)=2^{p}-1 .
$$

An argument similar to that in the previous cases shows that

$$
q^{r}-1>\left(q^{r}+1\right) /(q+1)=2^{p}-1,
$$

and so $q^{r}>2^{p}$. Let $q=t^{f}$, where $t$ is a prime and $f$ is a natural number. We now consider three cases separately.

(i) $r \geqslant 7$. Then $q^{r(r+1) / 2}>q^{3(r+1)} \geqslant 2^{3} q^{3 r}>2^{3(p+1)}$, which forces by Lemma 2.9 that $t=2$. Thus $\left(2^{f r}+1\right) /\left(2^{f}+1\right)=2^{p}-1$, and, consequently, $f=1$, $r=3$ and $p=2$, which is a contradiction.

(ii) If $r=5$, then $\left(q^{5}+1\right) /(q+1)=2^{p}-1$. Arguing as in the case $(i)$, we conclude that $t=2$ and $f=1$, whence $12=2^{p}$, a contradiction.

(iii) If $r=3$, then $\left(q^{3}+1\right) /(q+1)=2^{p}-1$. It follows that $q(q-1)=2\left(2^{p-1}-1\right)$, and so $q=p=2$, which is impossible.

Step 3.31. $K / H \oiint^{2} A_{r-1}(q)$.

In this case, we have

$$
|K / H|=\left.\right|^{2} A_{r-1}(q) \mid=\frac{1}{(r, q+1)} q^{r(r-1) / 2} \prod_{i=2}^{r}\left(q^{i}-(-1)^{i}\right),
$$

and the only odd order component of ${ }^{2} A_{r-1}(q)$ is $\left(q^{r}+1\right) /(q+1)(r, q+1)$. Thus

$$
\frac{q^{r}+1}{(q+1)(r, q+1)}=2^{p}-1,
$$

As before, we deduce that $q^{r} \geqslant 2^{p}$. Let $q=t^{f}$, where $t$ is a prime and $f$ is a natural number. We now consider three cases separately.

(i) $r \geqslant 7$. It follows that $q^{r(r-1) / 2} \geqslant q^{3 r}>2^{3 p}$, which implies that $t=2$ by Lemma 2.9. Now, we obtain

$$
\frac{2^{f r}+1}{\left(2^{f}+1\right)\left(r, 2^{f}+1\right)}=2^{p}-1,
$$

which contradicts Lemma 2.1 because $2^{p}-1$ is the largest prime in $\pi(G)$. 
(ii) $r=5$. In this case we have $q^{5}+1=(q+1)\left(2^{p}-1\right)(5, q+1)$. Assume first that $q$ is even, that is $q=2^{f}$. If $(5, q+1)=1$, then we obtain $2^{5 f}=2^{f+p}+2^{p}-2^{f}-2$, which is impossible. If $(5, q+1)=5$, then $2^{5 f}=5\left(2^{f+p}+2^{p}-2^{f}\right)-6$, which is again a contradiction. Assume next that $q$ is odd. Noting that $q(q-1)\left(q^{2}+1\right)=\left(2^{p}-1\right)(5, q+1)-1$, it is easily seen that the left hand side is congruent to $0(\bmod 4)$, while the right hand side is congruent to $2(\bmod 4)$, a contradiction.

(iii) $r=3$. In this case, we have $\left(q^{3}+1\right) /(q+1)(3, q+1)=2^{p}-1$. If $(3, q+1)=1$, then we obtain

$$
q(q-1)=2^{p}-2=2\left(2^{(p-1) / 2}-1\right)\left(2^{(p-1) / 2}+1\right) .
$$

If $q$ divides 2 , than $p=2$, a contradiction. If $q$ divides $2^{(p-1) / 2}-1$ or $2^{(p-1) / 2}+1$, then

$$
q(q-1)<2^{p}-2=2\left(2^{(p-1) / 2}-1\right)\left(2^{(p-1) / 2}+1\right),
$$

a contradiction. Therefore we may assume that $(3, q+1)=3$. If $q$ is even, then we conclude that $q=4$, which is a contradiction. We now suppose that $q$ is odd. Since $q(q-1)=2^{2}\left(3 \cdot 2^{p-2}-1\right)$, it follows that $(q-1)_{2}=4$, and so $(q+1)_{2}=2$. Moreover, under these hypotheses, one can easily deduce that $f$ is odd, otherwise $8 \mid q-1=t^{f}-1=\left(t^{f / 2}-1\right)\left(t^{f / 2}+1\right)$, which is a contradiction. On the other hand, $|G / K|$ divides $f(3, q+1)$ and since $f$ is odd, $|G / K|_{2}=1$. Therefore a Sylow 2-subgroup of $H$ has order $2^{p^{2}-4}$. Again, using Lemma 2.5 , we see that $2^{p}-1 \mid 2^{p^{2}-4}-1$, which implies that $p=2$. This is a contradiction.

Step 3.32. $K / H \nsubseteq C_{r}(2)$.

The only odd order component of $C_{r}(2)$ is $2^{r}-1$. Thus $2^{r}-1=2^{p}-1$. It follows that $r=p, G / K=1$ and $H=1$, which means $G \cong C$. This completes the proof of the theorem.

Acknowledgment. We would like to thank Professor Arturo Magidin and the referee for the careful reading of this manuscript and for many insightful suggestions and remarks.

\section{References}

[1] M. Akbari and A. R. Moghaddamfar, Simple groups which are 2-fold ODcharacterizable, Bull. Malays. Math. Sci. Soc., 35(1) (2012), 65-77.

[2] R. W. Carter, Simple groups of Lie type, Wiley, London, 1972. 
[3] G. Y. Chen, A new characterization of sporadic simple groups, Algebra Colloq., 3(1) (1996), 49-58.

[4] A. S. Kondratév, On prime graph components of finite simple groups, Mat. Sb., 180(6) (1989), 787-797.

[5] P. Mihăilescu, Primary cyclotomic units and a proof of Catalan's conjecture, J. Reine Angew. Math., 572 (2004), 167-195.

[6] A. R. Moghaddamfar, Recognizability of finite groups by order and degree pattern, Proceedings of the International Conference on Algebra 2010, 422-433.

[7] A. R. Moghaddamfar, A comparison of the order components in Frobenius and 2-Frobenius groups with finite simple groups, Taiwanese J. Math., 13(1) (2009), $67-89$.

[8] A. R. Moghaddamfar and S. Rahbariyan, OD-Characterization of some projective special linear groups over the binary field and their automorphism groups, Comm. Algebra (to appear).

[9] A. R. Moghaddamfar, A. R. Zokayi and M. R. Darafsheh, A characterization of finite simple groups by the degrees of vertices of their prime graphs, Algebra Colloq., 12(3) (2005), 431-442.

[10] H. G. Shi and G. Y. Chen, ${ }^{2} D_{p+1}(2)\left(5 \leqslant p \neq 2^{m}-1\right)$ can be characterized by its order components, Kumamoto J. Math., 18 (2005), 1-8.

[11] A. V. Vasilév and E. P. Vdovin, An adjacency criterion in the prime graph of a finite simple group, Algebra Logic, 44(6) (2005), 381-406.

[12] A. V. Vasilév and E. P. Vdovin, Cocliques of maximal size in the prime graph of a finite simple group, Algebra Logic, 50(4) (2011), 291-322.

[13] J. S. Williams, Prime graph components of finite groups, J. Algebra, 69(2) (1981), 487-513.

[14] K. Zsigmondy, Zur theorie der potenzreste, Monatsh. Math. Phys., 3(1) (1892), 265-284.

M. Akbari and A. R. Moghaddamfar

Department of Mathematics,

K. N. Toosi University of Technology,

P. O. Box 16315-1618,

Tehran, Iran

e-mails: m.akbari@dena.kntu.ac.ir (M. Akbari)

moghadam@kntu.ac.ir; moghadam@ipm.ir (A. R. Moghaddamfar) 